\title{
Suppression of endoplasmic reticulum stress-induced invasion and migration of breast cancer cells through the downregulation of heparanase
}

\author{
YANG $\mathrm{LI}^{1}$, HAO LIU ${ }^{1 *}$, YING YING HUANG ${ }^{1}$, LONG JIAN PU ${ }^{1}$, XU DONG ZHANG ${ }^{1,2}$, \\ CHEN CHEN JIANG ${ }^{1,2^{*}}$ and ZHI WEN JIANG ${ }^{1}$ \\ ${ }^{1}$ Faculty of Pharmacy, Bengbu Medical College, Bengbu, Anhui 233000, P.R. China; \\ ${ }^{2}$ Immunology and Oncology Unit, Calvary Mater Newcastle Hospital, Newcastle, NSW 2300, Australia
}

Received December 10, 2012; Accepted February 11, 2013

DOI: 10.3892/ijmm.2013.1292

\begin{abstract}
Tumor metastasis is the ultimate stage of cancer, and the primary cause of mortality in patients. Tumor cells breaking through the natural barrier consisting of the basement membrane (BM) and extracellular matrix (ECM) is the a crucial step in tumor invasion and metastasis. Thus, protecting this barrier is the key to reducing mortality. Heparanase is a mammalian endo- $\beta$-glucuronidase which has been found to promote the cleavage of heparan sulfate (HS), and plays a significant role in tumor cell invasion and metastasis. Although chemotherapeutic reagents have a strong antitumor activity, they may promote the invasion and migration of cancer cells, as has been observed during clinical treatment. Chemotherapeutic reagents can induce endoplasmic reticulum (ER) stress; in this study, we used adriamycin (ADM) and a classical ER stress inducer, tunicamycin (TM). We report that the activation of ER stress is involved in the enhanced invasion and migration ability of breast cancer cells and we hypothesized that this effect is associated with the activation of heparanase. In support of this, we used the heparanase inhibitor, OGT2115, and low molecular weight heparin (LMWH) to inhibit the expression and activity of heparanase, and we found that the invasion and migration ability of the cells was suppressed. Our findings demonstrate
\end{abstract}

Correspondence to: Professor Hao Liu, Faculty of Pharmacy, Bengbu Medical College, 2600 Donghai Street, Bengbu, Anhui 233000, P.R. China

E-mail: liuhao6886@yahoo.com.cn

Professor Chen Chen Jiang, Immunology and Oncology Unit, Room 443, David Maddison Clinical Sciences Building, Corner King and Watt Streets, Calvary Mater Newcastle Hospital, Newcastle, NSW 2300, Australia

E-mail: chenchen_jiang@yahoo.com.cn

*Contributed equally

Key words: breast cancer, invasion, migration, endoplasmic reticulum stress, chemotherapeutic reagents, heparanase that heparanase inhibitors suppress breast cancer cell invasion and migration induced by ER stress, and provide a strong rationale for the development of heparanase-based therapeutics for the prevention of metastasis induced by chemotherapeutic reagents.

\section{Introduction}

Breast cancer is the most common malignancy diagnosed among women worldwide; in the United States (1), more than 1 million new cases of breast cancer were diagnosed in 2010 (2). The majority of breast cancer-related deaths are due to the development of distant metastases, for which there are no effective treatments $(3,4)$. Although many chemotherapeutic reagents are available for the treatment of cancer metastasis, the median survival duration has not improved, and the molecular events that are associated with disease progression to metastasis are not yet fully understood (5). Tumor invasion and metastasis is an integrated process; intriguingly, chemotherapeutic reagents may be one of the many contributors to cancer metastasis, and it is believed that occurs through the activation of endoplasmic reticulum (ER) stress and heparanase by these agents (6).

Heparan sulfate (HS) proteoglycan (HSPG) (7) is an important component of the extracellular matrix (ECM) and basement membrane (BM). The degradation of HSPG is achieved through the cleavage of a glycosidic bond by heparanase, using a hydrolase mechanism. In addition, heparanase plays a significant role in cancer metastasis and invasion (8). Heparanase can be regulated by glucose, promoter methylation, p53, estrogen, tumor necrosis factor- $\alpha$ and interferon- $\gamma$ (9-11). Its HS degradation activity can be inhibited by the heparanase inhibitors, OGT2115, and low molecular weight heparin (LMWH) (12,13).

Chemotherapeutic reagents have been shown to induce ER stress. In this study, we used two reagents to induce ER stress, the chemotherapeutic drug, adriamycin (ADM), and the ER stress inducer, tunicamycin (TM). Invasion and metastasis appear during the long-term cancer treatment process, prompting us to speculate that these events may be associated with the increased ER stress. A number of factors, such as hypoxia, nutritional deficiency, oxidative stress, chemo- and 
radiotherapy, calcium metabolism disorders, and defects in protein expression can cause ER stress $(14,15)$. The ER responds to stress conditions by activating a range of stress response signaling pathways to alter transcriptional and translational programs, which couple the ER protein folding load with the ER protein folding capacity. This process is termed the unfolded protein response (UPR) (16) and the marker protein is glucose-regulated protein 78 (GRP78). UPR can protect the ER and minimize damage to other organelles, and it may protect cells by promoting metastasis (17).

The aim of this study was to investigate the correlation between ER stress and the increased invasion and migration of cancer cells. Furthermore, we provide evidence that the invasion and migration induced by chemotherapeutic reagents occurs due to the activation of heparanase under ER stress.

\section{Materials and methods}

Reagents and antibodies. TM was purchased from Sigma Chemical Co. (Castle Hill, NSW, Australia). OGT2115 was purchased from Tocris Bioscience (Bristol, UK). ADM was purchased from Pharmacia \& Upjohn SpA and LMWH was purchased from Sanofi-Aventis Pharmaceutical Co., Ltd. (Beijing, China), for clinical use. The rabbit monoclonal antibody (mAb) against GRP78, heparanase, and $\beta$-actin antibody were purchased from Santa Cruz Biotechnology, Inc. (Santa Cruz, CA, USA). Human heparanase enzyme-linked immunosorbent assay (ELISA) kit was obtained from R\&D Systems (Minneapolis, MN, USA). Matrigel was purchased from BD Biosciences (Bedford, MA, USA). The 24-well Transwell insert $(8 \mu \mathrm{m})$ was obtained from Corning Inc. (Corning, NY, USA). 3-(4,5-Dimethylthiazol-2-yl)-2,5-diphenyltetrazolium bromide (MTT) was purchased from Sigma Chemical Co. Dulbecco's modified Eagle's medium (DMEM), fetal bovine serum (FBS) and phosphate-buffered saline (PBS) were purchased from Gibco (Grand Island, NY, USA).

Cell lines. The breast cancer cell lines, MDA-MB-231 and MDA-MB-435, were obtained from the American Type Culture Collection (ATCC). Cells were routinely cultured in DMEM supplemented with $10 \%$ FBS and $100 \mathrm{U}$ of penicillin-streptomycin with $5 \% \mathrm{CO}_{2}$ in a humidified incubator at $37^{\circ} \mathrm{C}$. All cell lines were tested every month for mycoplasma contamination, used only at low passage, and were regularly examined under a microscope for phenotypic changes prior to use.

Cell viability assay. The cytotoxic effect of OGT2115 and ADM on breast cancer cells was determined using the MTT assay as previously described. MTT is a yellow tetrazolium dye that responds to metabolic activity. Reductase enzymes in living cells reduce MTT from a pale yellow color to dark blue formazan crystals. Cells were plated at 7,000/well in 96-well plates and cultured in a humidified $5 \% \mathrm{CO}_{2}$ atmosphere at $37^{\circ} \mathrm{C}$. At 24,48 and $72 \mathrm{~h}$, the wells were incubated with MTT $(5 \mathrm{mg} / \mathrm{ml})$ in PBS for $4 \mathrm{~h}$ at $37^{\circ} \mathrm{C}$. After $4 \mathrm{~h}$, the MTT solution was removed and replaced with $150 \mu \mathrm{l}$ of dimethyl sulfoxide (DMSO). The plate was further incubated for $0.5 \mathrm{~h}$ at room temperature, and the optical density (OD) of the wells were determined using a plate reader at a test wavelength of $570 \mathrm{~nm}$. Each test was performed in triplicate.
Cell invasion assay. The invasion assay was performed using a 24-well cell culture plate with $8.0-\mu \mathrm{m}$ pore membrane inserts. Breast cancer cells were starved in serum-free medium overnight, and $5 \times 10^{4}$ cells were resuspended in $100 \mu \mathrm{l}$ serum-free medium and placed in the upper chambers. The membrane undersurface was coated with $50 \mu$ l Matrigel from BD Biosciences mixed with RPMI-DMEM serum free medium at a $1: 8$ dilution for $30 \mathrm{~min}$ at $37^{\circ} \mathrm{C}$. The lower well of each chamber was filled with $600 \mu 1$ DMEM supplemented with $10 \%$ FBS and incubated for $48 \mathrm{~h}$. Reagents were added to the upper chambers, and $48 \mathrm{~h}$ after treatment, the cells on the upper surface of the membrane were removed by cotton buds, and the cells on the lower chamber were incubated with paraformaldehyde in PBS buffer and stained with $0.1 \%$ crystal violet. Five visual fields were randomly selected for each insert and photographed under a light microscope at x400 magnification. The number of cells was then counted and analyzed for statistically significant differences. Each condition was assayed in triplicate, the experiments were performed independently at least three times, and the results are expressed as the number of cells/field. A one-way analysis of variance was used to determine statistical significance.

Cell migration assay. Migration assay was performed using a 24-well cell culture plate with $8.0-\mu \mathrm{m}$ pore membrane inserts without Matrigel. MDA-MB-435 and MDA-MB-231 cells $\left(5 \times 10^{4}\right)$ were added to the upper wells, and the chambers were incubated for $24 \mathrm{~h}$ at $37^{\circ} \mathrm{C}$. The lower chamber was filled with $600 \mu 110 \%$ FBS as the chemoattractant. After $24 \mathrm{~h}$ in normoxic conditions the cells that had migrated were stained and photographed under a light microscope at x200 magnification. The number of cells that had migrated was counted from five randomly selected fields. Each condition was assayed in triplicate, the experiments were performed independently at least three times, and the results are expressed as the number of cells/field. A one-way analysis of variance was used to determine statistical significance.

Wound healing assay. Cells were plated on six-well plates at $5 \times 10^{5}$ cells/well. The following day, the cells were washed with PBS and wounds were created by scraping with a sterilized pipette tip. The cells were then washed twice with PBS, and incubated in RPMI-DMEM. The wound closure was monitored for 0-48 h. The wound areas were observed under an inverted microscope and measured by imaging at the relevant fields for the calculation of the healing percentages. Each test was performed in triplicate.

Western blot analysis. Cells were washed three times with cold PBS and lysed on ice in radioimmunoprecipitation assay (RIPA) buffer with protease inhibitors. The protein concentrations were determined using the BCA method. A total of $80 \mu \mathrm{g}$ of protein was separated by $10 \%$ SDS-PAGE and electroblotted onto PVDF membranes using a semi-dry blotting apparatus. After blocking in 5\% non-fat milk, the membranes were incubated overnight at $4^{\circ} \mathrm{C}$ with the primary antibodies. The membranes were then incubated in the secondary antibodies for $2 \mathrm{~h}$ at room temperature on a shaker. The bands were visualized using Western Lightning ECL Pro with horseradish peroxidase (HRP). $\beta$-actin was used as a loading control. 


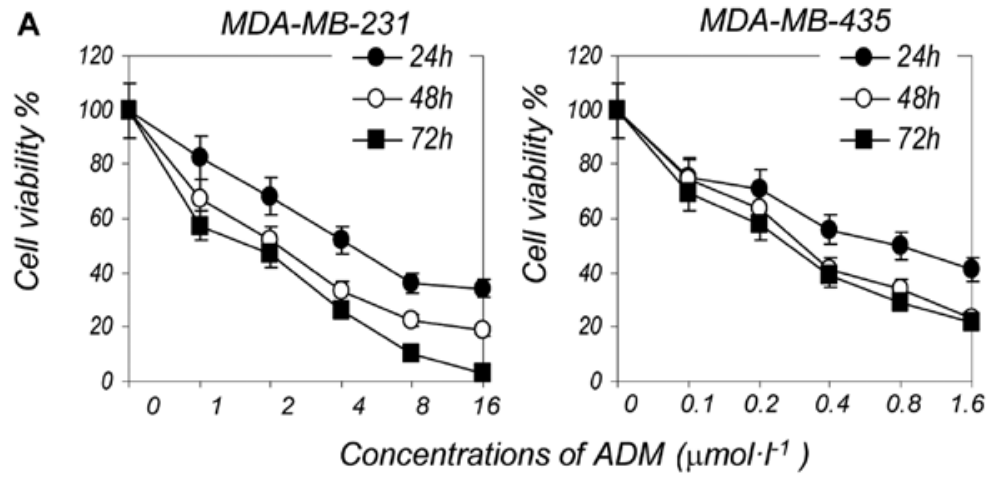

B
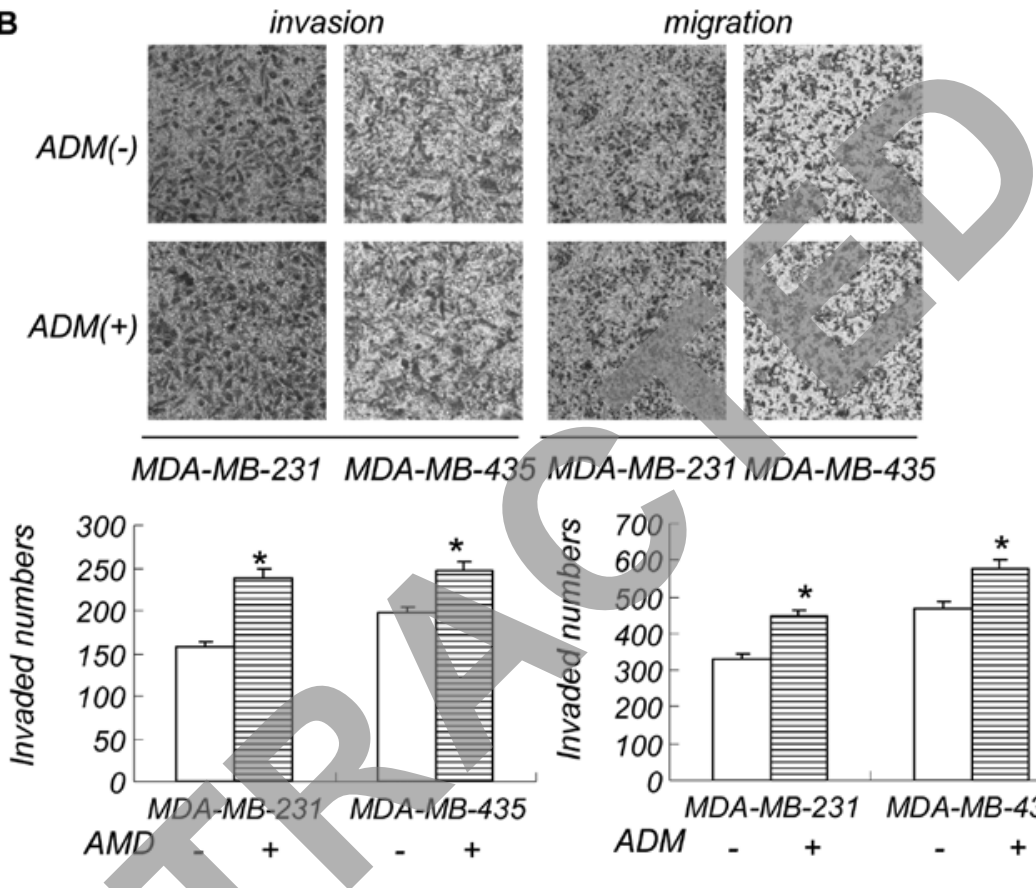

Figure 1. A low concentration of adriamycin (ADM) increases the invasion and migration of breast cancer cells. (A) ADM significantly inhibited the growth of breast cancer cells. ADM decreased the viability of breast cancer cells as reflected by the reduction of the MTT signal measured colorimetrically by the absorbance of the formazan product. Breast cancer cells (MDA-MB-231 and MDA-MB-435) were treated with ADM at various concentrations and measured after three days in culture. Concentrations used ranged from 0.1 to $1.6 \mu \mathrm{M}$ in MDA-MB-435 cells, and ranged from 1 to $16 \mu \mathrm{M}$ in MDA-MB-231 cells. The $\mathrm{IC}_{50}$ was $0.6 \mu \mathrm{M}$ in the MDA-MB-435, and $1 \mu \mathrm{M}$ in the MDA-MB-231 cells. The results are expressed as a percentage of the control levels. Data are presented as the means \pm SEM, $n=3, " P<0.05$ compared to the controls. (B) The invasion and migration of breast cancer cells were increased following exposure to a low concentrations of ADM. A low concentration of ADM $(0.2 \mu \mathrm{M})$ increased cell invasion and migration in MDA-MB-435 and MDA-MB-231 cells. Using ADM untreated cells as the controls, five visual fields were randomly selected for each insert and photographed under a light microscope at $\mathrm{x} 400$ and $\mathrm{x} 200 \mathrm{magnifica-}$ tion. The significance in the differences in cell number was analyzed, ${ }^{*} \mathrm{P}<0.05$ compared to the controls.

ELISA. Utilizing the ELISA method for the detection and quantification of heparanase, we were able to monitor changes in heparanase activity. Cells were plated on 24-well plates at $5 \times 10^{4} /$ well and incubated in DMEM containing 10\% FBS. After $24 \mathrm{~h}$, we extracted the cell culture medium for the detection of heparanase activity. The microtiter plate provided with the kit was pre-coated with an antibody specific to heparanase. Standards or samples were then added to the appropriate microtiter plate wells containing a biotin-conjugated polyclonal antibody to heparanase. Avidin conjugated to HRP was then added to each microplate well and incubated. Tetramethylbenzidine (TMB) substrate solution was then added to each well. Only those wells that contained heparanase, biotin-conjugated antibody and enzyme-conjugated avidin exhibited a change in color. The enzyme-substrate reaction was terminated by the addition of sulphuric acid solution and the color change was measured spectrophotometrically at a wavelength of $450 \pm 2 \mathrm{~nm}$. The concentration of heparanase in the samples was then determined by comparing the OD.

\section{Results}

A low concentration of ADM increases the invasion and migration ability of breast cancer cells. We first examined the effect of ADM on the viability of the breast cancer cell lines, MDA-MB-231 and MDA-MB-435. ADM significantly inhibited the growth of MDA-MB-231 cells at an $\mathrm{IC}_{50}$ of $1 \mu \mathrm{M}$. The $\mathrm{IC}_{50}$ of ADM was $0.6 \mu \mathrm{M}$ in the MDA-MB-435 cells (Fig. 1A). We examined the effects of various concentrations of ADM on cell invasion and migration, and found the $\mathrm{IC}_{50}$ of $\mathrm{ADM}$ had almost no effect on cell invasion and migration (data not shown). However, a low concentration of ADM $(0.2 \mu \mathrm{M})$ did not have a significant effect on cell death, but increased cell invasion and migration to a certain extent (Fig. 1B). 


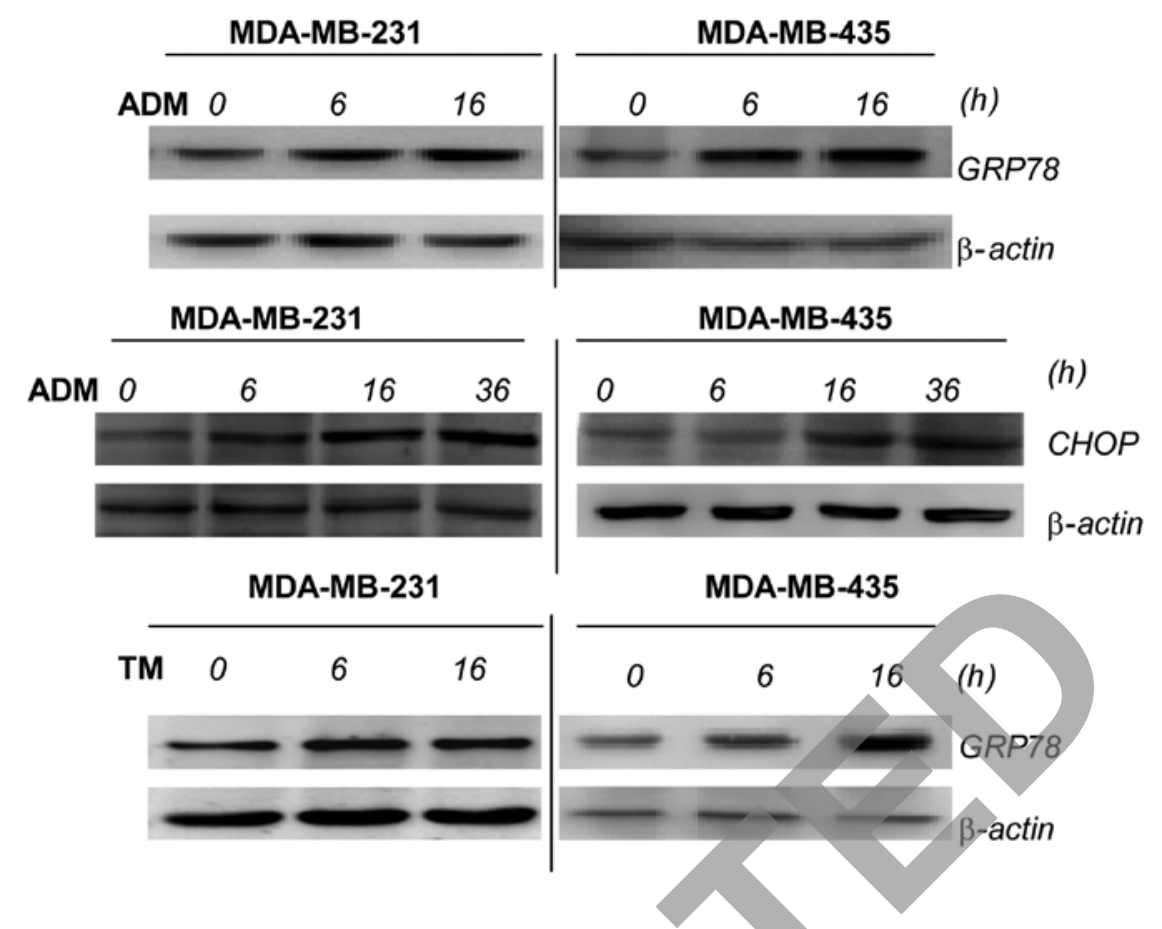

Figure 2. Tunicamycin (TM) and adriamycin (ADM) induce ER stress in breast cancer cells. Breast cancer cells (MDA-MB-231 and MDA-MB-435) were treated with TM at $0.75 \mu \mathrm{M}$ and ADM at $0.6 \mu \mathrm{M}$, and after 6 and $16 \mathrm{~h}$, cell lysates were prepared and examined by western blot analysis. $\beta$-actin was used as a loading control. The expression of GRP78 and CHOP significantly increased in the MDA-MB-231 and MDA-MB-435 cells following treatment with TM or ADM.

Consistent with cancer metastasis data in clinical practice, a high concentration of ADM suppressed cell proliferation, but a low concentration induced breast cancer cell metastasis. A low degree of ER stress can protect cells but induces apoptosis when the ER response is strong enough. Metastasis is likely associated with the induction of ER stress by low concentrations of chemotherapeutic reagents, which can protect cancer cells. In order to verify our assumption, we conducted the following experiments.

TM and ADM induce ER stress in breast cancer cells. ADM is a chemotherapeutic reagent which can induce ER stress. Using ADM, we examined whether the increase in the invasion and migration of breast cancer cells is due to the induction of ER stress. To monitor ER stress induction, we detected the expression of GRP78 and C/EBP homologous protein (CHOP) in the breast cancer cells following treatment with ADM by western blot analysis. GRP78 is an indicator of ER stress, and ER stress transducers are kept in an inactive state through binding to the ER chaperone, GRP78 (18); treatment with ADM increases the levels of GRP78 and CHOP. To further verify that the increase in invasion and migration is indeed caused by ER stress, we used the ER stress inducer, TM. The results showed that the cells exposed to TM expressed higher levels of GRP78 (Fig. 2).

ER stress activates heparanase in breast cancer cells. We then exposed the breast cancer cells to TM, and consistent with our findings using ADM, a low concentration of TM also increased cell invasion and migration. The number of cells that underwent invasion and migration, and the speed of this process are shown in Figs. 3A and 4D. Since TM is an ER stress inducer, combined with the ADM results, we believe that the invasion and metastasis observed is associated with ER stress. Heparanase plays a major role in tumor metastasis, and to determine whether ER stress induces heparanase activation in breast cancer cells, we performed western blot analysis and ELISA to detect the expression and activity of heparanase (Fig. 3B). The western blot analysis results revealed a change in the bands from 50 to $65 \mathrm{kDa}$, indicating the activation of heparanase. In addition, the increased signal shown by ELISA also reflected changes in heparanase activity. The results demonstrate that the ER stress inducer, TM, activates heparanase in breast cancer cells. Heparanase activity increased at 16 and $24 \mathrm{~h}$, causing a series of after-effects and cell invasion and migration.

Heparanase inhibitor decreases the invasion and migration induced by ER stress. As heparanase promotes tumor cell invasion and migration, we then examined whether the ER stress-induced cell invasion and migration occurs through the induction of heparanase. In order to prove that heparanase plays a decisive role in enhancing cell invasion under ER stress, we used OGT2115 to inhibit heparanase activity. OGT2115 is a heparanase inhibitor that exhibits anti-angiogenic properties in vitro by directly suppressing heparanase activity. First, we determined whether OGT2115 can inhibit heparanase. Our ELISA results confirmed that OGT2115 suppressed heparanase activity (Fig. 4A). Since TM enhances cell invasion and migration when administered at a low concentration, we then examined whether OGT2115 can alter the effects of TM on cell invasion and migration. OGT2115 suppressed the invasion and migration of breast cancer cells, although not significantly (Fig.4B). However, compared with the control group, the number and rate of migrated cells were significantly reduced following the exposure of the cells to TM + OGT2115. OGT2115 signifi- 
A

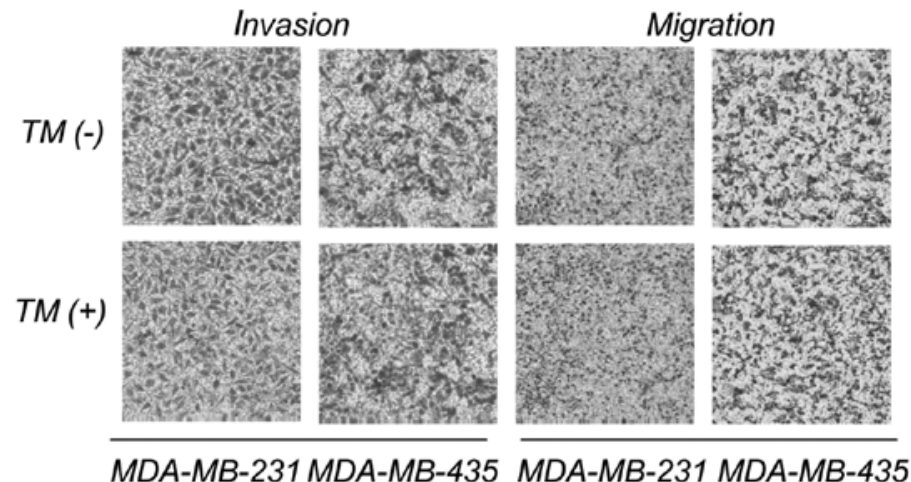

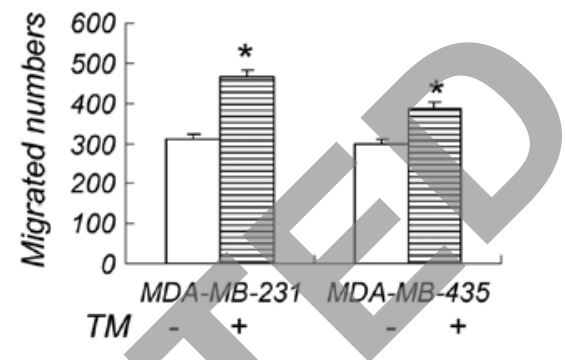

B

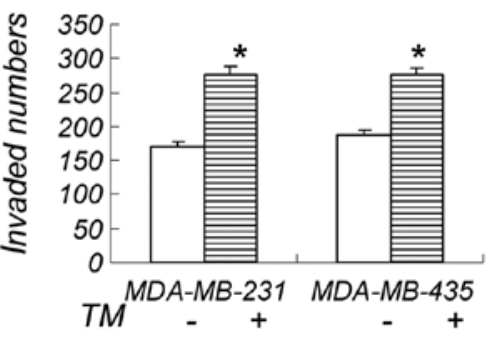

$M D A-M B-231$

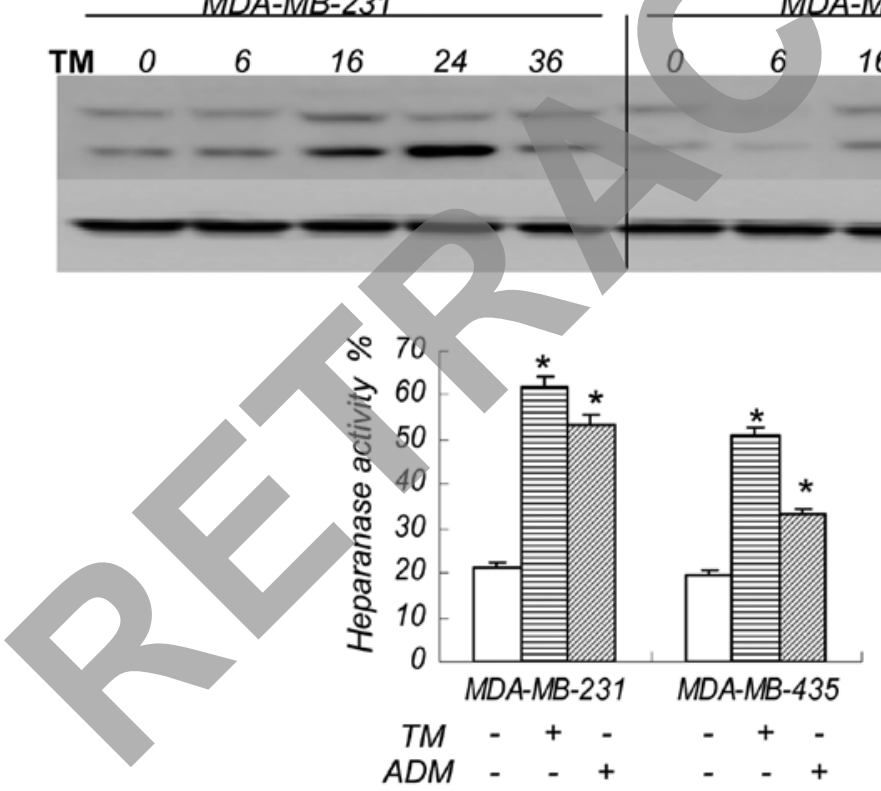

Figure 3. ER stress activates heparanase in breast cancer cells. (A) Tunicamycin (TM) induced an increase in the migration and invasion of cells. Breast cancer cells (MDA-MB-231 and MDA-MB-435) were treated with a low concentration of TM $(0.75 \mu \mathrm{M})$ and after 24 and $48 \mathrm{~h}$, TM significantly increased the number of migrated and invaded cells. * $\mathrm{P}<0.05$ compared to the controls. (B) Activation of heparanase under ER stress. The two cell lines were treated with TM at $0.75 \mu \mathrm{M}$, and after $36 \mathrm{~h}$, cell lysates were prepared and examined by western blot analysis. $\beta$-actin was used as a loading control. We used adriamycin (ADM) at $0.2 \mu \mathrm{M}$ and TM at $0.75 \mu \mathrm{M}$ in the two cell lines, and after $24 \mathrm{~h}$, the cell lysates were prepared and examined by the ELISA. Bands and the percentage of enzyme activity reflect the extent of the activation of heparanase. Hpa, heparanase.

cantly inhibited the invasion and migration induced by ADM (Fig. 4C and D). Furthermore, the MTT assay results showed that OGT2115 did not decrease the anti-proliferative effect of ADM, thus preserving the strong antitumor activity of the chemotherapeutic drug (Fig. 4E).

LMWH decreases the invasion and migration induced by ER stress. In order to validate the above results, we also selected another heparanase inhibitor in the following experiments. LMWH as an exogenous supplement of heparins is susceptible to cleavage by heparanase in vitro, and this cleavage significantly neutralizes the anti-coagulant properties of these polysaccharides (19). LMWH exhibited a moderate antitumor activity and decreased the heparanase activity induced by ADM or TM (Fig.5A and B).However, it significantly reduced cell invasion and migration when used in combination with TM (Fig. 5C and 4D). 
A

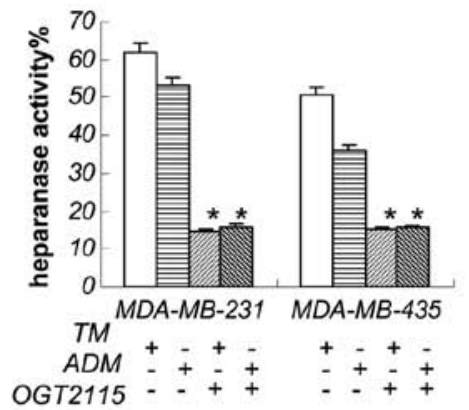

B

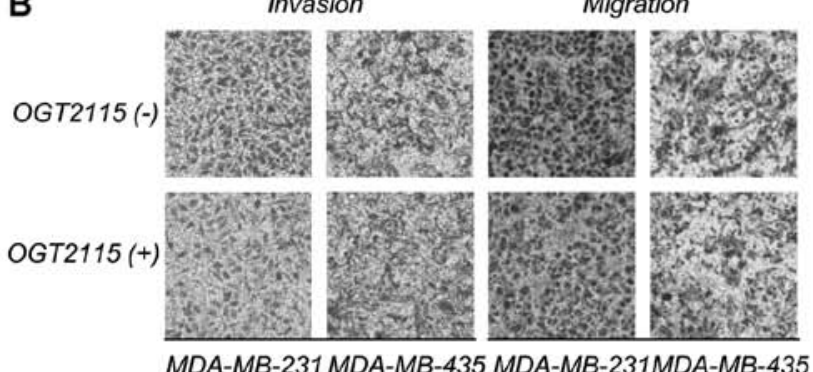

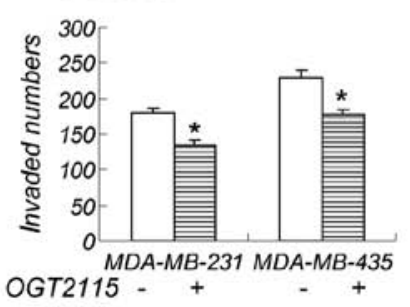
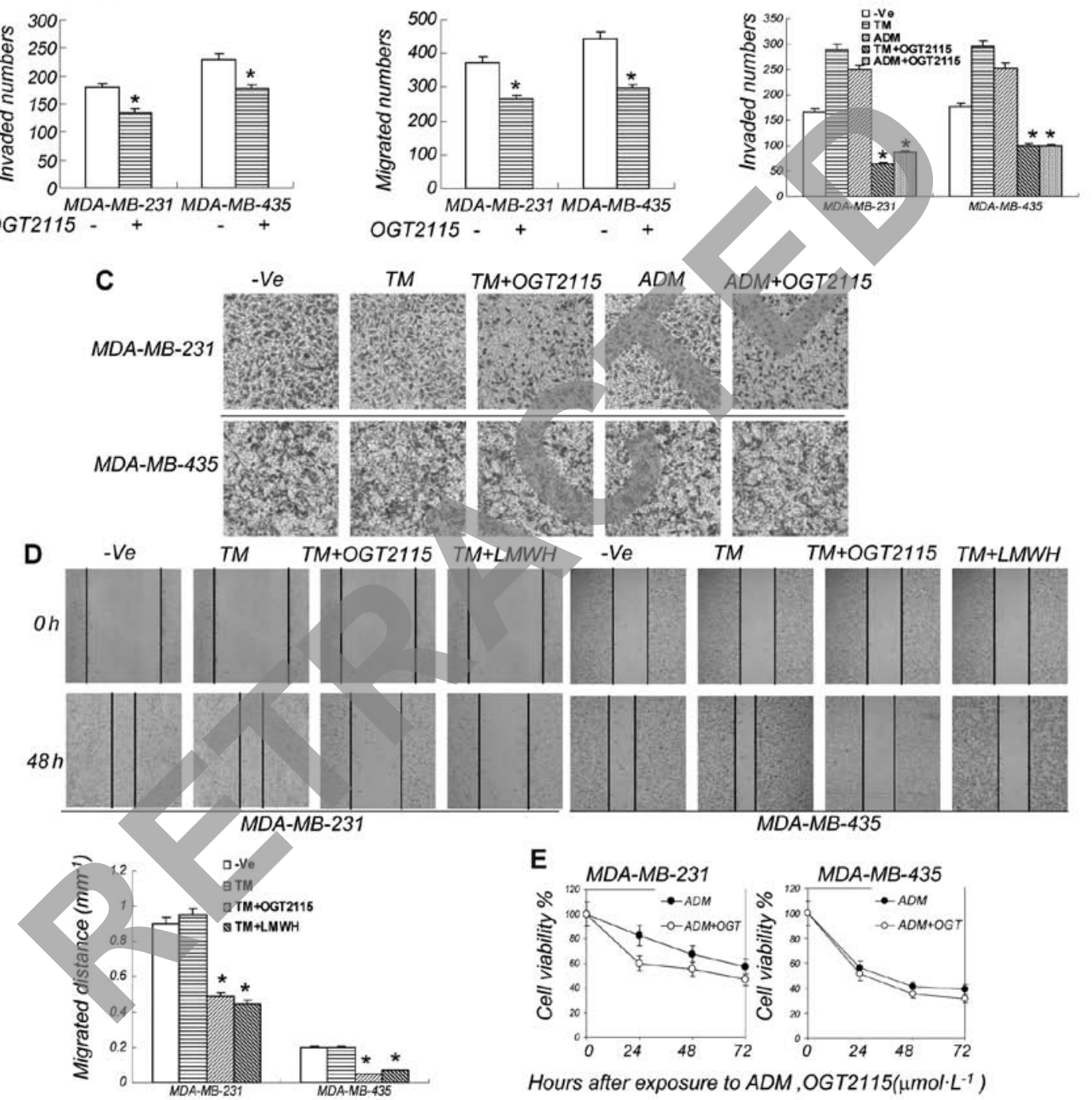

Figure 4. Heparanase inhibitor decreases the invasion and migration induced by ER stress. (A) OGT2115 suppressed the heparanase activity induced by ER stress. The breast cancer cell lines, MDA-MB-231 and MDA-MB-435, were treated for $24 \mathrm{~h}$ with tunicamycin (TM) at $0.75 \mu \mathrm{M}, \mathrm{ADM}$ at $0.2 \mu \mathrm{M}$ and OGT2115 at $0.8 \mu \mathrm{M}$. Cell lysates were then prepared and examined for heparanase enzyme activity. (B) Effect of OGT2115 on the invasion of breast cancer cells. Cells were treated with OGT2115 at $1.6 \mu \mathrm{M}$ for 24 and $48 \mathrm{~h}$; the results showed that OGT2115 decreased the numbers of cells undergoing invasion and migration. "P<0.05 compared to the controls. (C) Effect of TM/adriamycin (ADM) and OGT2115 on the invasion of breast cancer cells. Cells were treated with TM at $0.75 \mu \mathrm{M}$ or ADM $0.2 \mu \mathrm{M}$ for after $48 \mathrm{~h}$; the results showed that TM and ADM increased the numbers of cells undergoing invasion. We then treated the cells with OGT2115 at $0.8 \mu \mathrm{M}$ to suppress this increased invasion, "P $<0.05$ compared to the controls. (D) Effect of TM/ADM and OGT2115 on the migration of breast cancer cells. Cells were treated with TM at $0.75 \mu \mathrm{M}$ or $\mathrm{ADM}$ at $0.2 \mu \mathrm{M}$ for $48 \mathrm{~h}$; the results showed that TM and ADM increased the number of cells undergoing migration and invasion. We then treated the cells with OGT2115 at $0.8 \mu \mathrm{M}$ to suppress this increased migration. "P<0.05 compared to the controls. (E) OGT2115 enhances the anti-proliferative effect of ADM. A reduction in the MTT signal was detected colorimetrically based on the absorbance of the formazan product. These cells were then treated with ADM at $0.6 \mu \mathrm{M}$ combined with OGT2115 at $0.8 \mu \mathrm{M}$, and the results are expressed as a percentage of the control levels. All cell cultures incubated with OGT2115 exhibited a significant decrease in cell viability. Data are presented as the means $\pm \mathrm{SEM}, \mathrm{n}=3$. ${ }^{*} \mathrm{P}<0.05$ compared to the controls. -Ve, vehicle control.

We used LMWH in combination with ADM, and similar to the results obtained from the combination of LMWH and TM,
LMWH significantly reduced the cell migration and invasion induced by ADM (Fig. 5D). The results of the migration of 
A

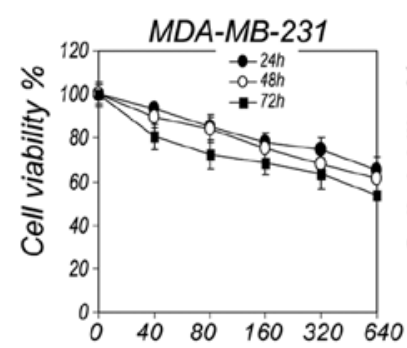

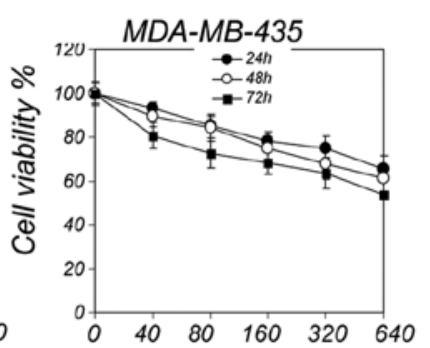

(Concentrations of $L M W H I U \cdot m r^{-1}$ )
B

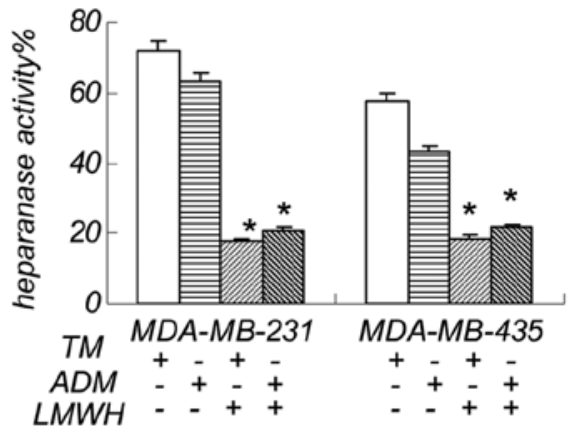

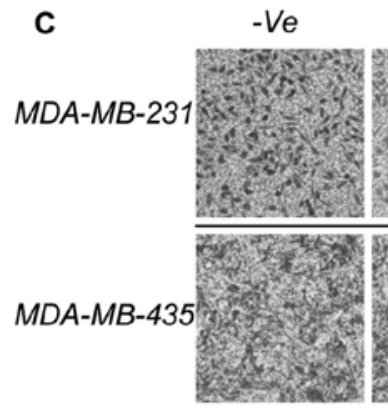

$T M$

$T M+L M W H \quad A D M$

$A D M+L M W H$
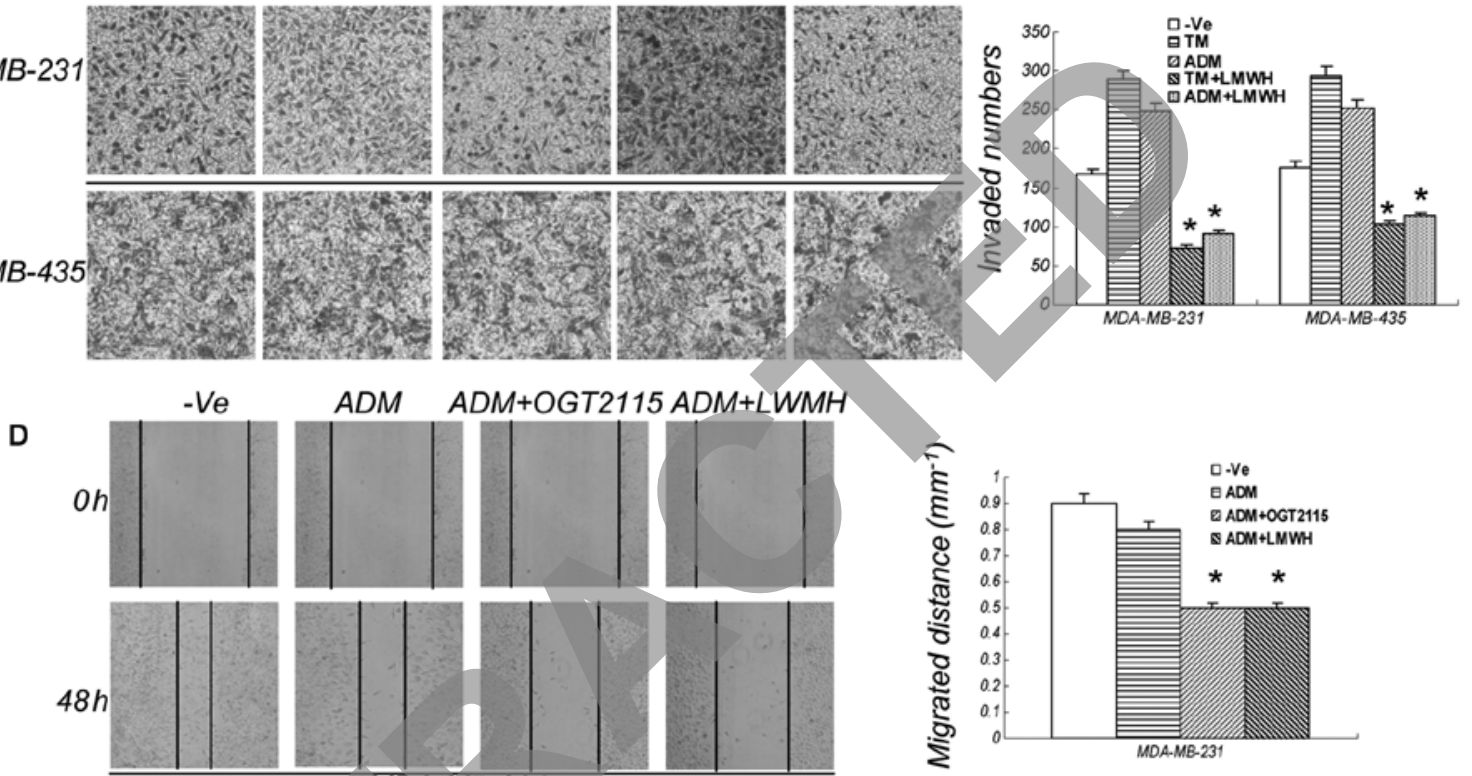

$A D M$ $A D M+O G T 2115 A D M+L W M H$
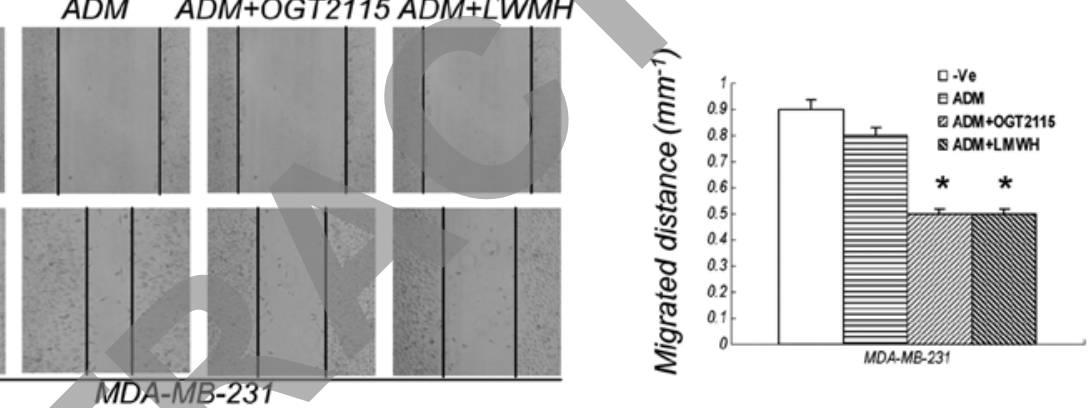

Figure 5. LMWH decreases the invasion and migration induced by ER stress. (A) LMWH moderately inhibited the growth of breast cancer cells. The breast cell lines, MDA-MB-435 and MDA-MB-231, were treated with LMWH at various concentrations and measured after three days in culture. The results are expressed as a percentage of the control levels. Data are presented as the means $\pm \mathrm{SEM}, \mathrm{n}=3$. ${ }^{*} \mathrm{P}<0.05$ compared to the controls. (B) LMWH suppressed the heparanase activity induced by ER stress. The breast cancer cell lines, MDA-MB-231 and MDA-MB-435, were treated for $24 \mathrm{~h}$ with TM at $0.75 \mu \mathrm{M}, \mathrm{ADM}$ at $0.2 \mu \mathrm{M}$ and LMWH at $500 \mathrm{IU} / \mathrm{ml}$. Cell lysates were then prepared and examined for heparanase enzyme activity. (C) Invasion of the cancer cells were decreased by LMWH. Following treatment with ADM at $0.2 \mu \mathrm{M}, \mathrm{TM}$ at $0.75 \mu \mathrm{M}$ and $\mathrm{LMWH}$ at $500 \mathrm{IU} / \mathrm{ml}$ for $48 \mathrm{~h}$, we observed that $\mathrm{LWMH}$ decreased the invasion induced by ADM or TM. "P $<0.05$ compared to the controls. - Ve, vehicle control. (D) Migration of the cancer cells were decreased by OGT2115/LMWH. Following treatment with ADM at $0.2 \mu \mathrm{M}$, OGT2115 at $0.8 \mu \mathrm{M}$ and LMWH at $500 \mathrm{IU} / \mathrm{ml}$ for $48 \mathrm{~h}$, we observed that OGT2115/LMWH decreased the migration induced by ADM. "P<0.05 compared to the controls. -Ve, vehicle control.

MDA-MB-435 cells are not shown. Thus, heparanase inhibitors play a significant role in decreasing cell invasion and migration induced by ER stress.

\section{Discussion}

In breast cancer, metastasis is an end result of a long selection process of clinical treatments spanning decades, in which the most adaptable cancer cells persist. More chemotherapeutic reagents, as well as radiation therapy are being included in cancer therapeutic regimens; however, in actual clinical practice, these reagents may increase the incidence of cancer cell metastasis (20). The reasons for the progression to metastasis for some patients during clinical treatment are unclear $(10,21)$. As reported in the literature, we know that heparanase is an important contributor to tumor invasion and metastasis (22). It has been reported that ionizing radiation promotes pancreatic cancer aggressiveness through the upregulation of heparanase expression (23). Our results suggest a correlation between ER stress-induced metastasis and heparanase.

HSPGs interact through specific attachment sites with the main protein components of BM and ECM, and only heparanase can degrade HSPG. This degradation is associated with the invasion, angiogenic and metastatic potential of diverse malignant tumors and cell lines. We used Matrigel cell invasion assay; Matrigel enables an environment conducive to cell invasion in vitro and the main ingredients of Matrigel are HSPG, laminin, collagen IV, nidogen and others. At room temperature, Matrigel automatically gathers to become a matrix material which is similar to the mammalian cell BM and ECM; it can produce the biological activity and analog cell BM structure in vivo. The expression of heparanase in tumor cells correlates with the increased metastatic potential (24). In addition, HS moieties in the ECM are responsible for the binding of heparin- 
binding growth factors, which are thereby protected, stabilized and sequestered from their site of action, but upon the enzymatic degradation of HS can be readily mobilized to induce growth factor-dependent processes. Thus, the cleavage of HS by heparanase enables cell invasion, the release of HS-bound angiogenic and growth factors from the ECM depots, and the generation of bioactive HS fragments which promote growth factor-receptor binding, dimerization and signaling $(25,26)$. Direct evidence for heparanase promoting the progression of many cancers is provided by the demonstration that the overexpression of heparanase accelerates primary tumor growth and increases the metastatic ability of melanoma and prostate carcinoma cells (27). By contrast, heparanase silencing markedly decreases the metastatic potential of cancer cells.

ER is a central organelle responsible for lipid synthesis, calcium homeostasis, protein folding and maturation. Previous studies have focused on the roles of ER stress in the inhibition of apoptosis and chemotherapy resistance in human cancers, and certain studies have reported that ER stress is involved in the regulation of tumor invasion and metastasis. However, it remains unclear whether ER stress is involved in the regulation of tumor invasion and metastasis (28). Only properly folded proteins are allowed to reach their final destination, whereas unfolded and misfolded proteins are exported or dislocated from the ER and degraded by cytoplasmic proteasomes (29). When the homeostasis of the ER is disturbed, unfolded or misfolded proteins accumulate in the ER lumen, resulting in ER stress. In response to ER stress, cells activate a set of tightly controlled regulatory programs, known as UPR, to restore the normal function of the ER. However, if ER stress is sustained and the adaptive UPR fails to eliminate unfolded or misfolded proteins, apoptosis will occur to remove the stressed cells. There are three branches of UPR that are initiated by distinct ER stress transducers located on the ER membrane: protein kinase RNA-like endoplasmic reticulum kinase (PERK), inositolrequiring enzyme-1 (IRE-1) (16) and activating transcription factor-6 (ATF-6) (30). All three ER stress transducers are kept in an inactive state through binding to the ER chaperone GRP78 (31), which is also known as immunoglobulin-binding protein. The exact mechanism underlying the switch of the UPR from a prosurvival mechanism to a proapoptotic response is unclear. Therefore, the UPR can be considered as a safeguard for protein synthesis, post-translational modifications, folding and secretion, calcium storage and signaling and lipid biosynthesis. The UPR initially tries to restore the normal function of the cell by halting protein translation and activating the signaling pathways that lead to the increase in the production of molecular chaperones involved in protein folding. If these objectives are not achieved within a certain period of time or the disruption is prolonged, the UPR tries to turn on the apoptotic pathway $(32,33)$. We demonstrated that chemotherapeutic reagents can promote ER stress and the activation of the UPR, which confers a survival advantage to the tumor cells, promoting their migration and invasion ability, and these effects are associated with the activation of heparanase.

We provide evidence that ER stress inducers can activate heparanase, and this activation results in the increased invasion and migration of breast cancer cells. The purpose of the UPR is to protect the ER and limit damage to other organelles, helping cells to leave the original stressed environment and thus enabling cells to survive. Our findings indicate that the heparanase inhibitor, OGT2115, and LMWH can suppress metastasis induced by ER stress in breast cancer cells. The degradation of LMWH by heparanase in vivo may be relevant in situations in which heparanase is overexpressed, and treatment with LMWH composed of non-anticoagulant species of heparin and various sulfated polysaccharides which inhibit experimental metastasis, also inhibited heparanase activity in the tumor cells (34). However, the precise molecular mechanisms responsible for heparanase regulation have not yet been fully elucidated. HSPG contains sulfate groups and a sugar chain and is negatively charged. These biological or chemical characteristics can inhibit metastasis. According to its basic chemical composition, heparanase inhibitors can be divided into sugars, nucleotides and amino acids, such as oligomannurarate sulfate (35), laminarin sulfate, phosphomannopentaose sulfate (36), LMWH and others. Heparanase inhibitors do not decrease the anti-proliferative effect of chemotherapeutic reagents, and they also inhibit the invasion and migration of cancer cells under ER stress. Our findings may prove to be clinically significant, since we show that $\mathrm{ER}$ stress is a pivotal contributor in chemotherapy-mediated tumor metastasis. In addition, since GRP78 and heparanase play roles in the chemotherapy-induced increase in invasion or migration, the mechanism behind the ER stress-induced invasion and migration may be through the activation of heparanase. However, the inhibition of heparanase activity did not completely suppress cell invasion, suggesting that other factors may also contribute to the ER stress-induced increase in cell invasion and metastasis following chemotherapy.

In conclusion, to our knowledge, we demonstrate for the first time in this study that ER stress increases the invasion and migration of breast cancer cells through the activation of heparanase. This may occur through the activation of the UPR which plays an important role in the protection of cells against the cytotoxic effects of low-dose chemotherapy. It is essential to elucidate the molecular mechanisms that underlie the increase in cancer metastasis induced by chemotherapy. Our results suggest that heparanase is involved in chemotherapyinduced tumor metastasis, and that inhibiting heparanase activity may prove to be a promising therapeutic strategy for the treatment of metastatic breast cancer. In our study, cell invasion and migration were suppressed by the inhibition of heparanase and this finding may have a significant impact on the development of heparanase-based therapy for metastasis under ER stress $(37,38)$.

\section{Acknowledgements}

This study was supported by grants from the National Natural Science Foundation of China (no. 81000992 and 81072207), the Natural Science Foundation of Anhui Province (no. 090413135) and the Key Project of the Natural Science Foundation of the Department of Education, Anhui Province, China (no. KJ2012A202).

\section{References}

1. Qin XJ and Ling BX: Proteomic studies in breast cancer (Review). Oncol Lett 3: 735-743, 2012. 
2. Spinelli GP, Russo LG, Miele E, et al: Breast cancer metastatic to the pituitary gland: a case report. World J Surg Oncol 10: 137, 2012.

3. Gnant M, Balic M, Petru E, et al: Treatment of bone metastases in patients with advanced breast cancer. Breast Care (Basel) 7: 92-98, 2012.

4. Breidenbach M, Rein DT, Schondorf T, et al: A new targeting approach for breast cancer gene therapy using the heparanase promoter. Cancer Lett 240: 114-122, 2006.

5. Coleman RE: Clinical features of metastatic bone disease and risk of skeletal morbidity. Clin Cancer Res 12: 6243-6249, 2006.

6. Fux L, Ilan N, Sanderson RD and Vlodavsky I: Heparanase: busy at the cell surface. Trends Biochem Sci 34: 511-519, 2009.

7. Vlodavsky I, Elkin M, Pappo O, et al: Mammalian heparanase as mediator of tumor metastasis and angiogenesis. Isr Med Assoc 2: 37-45, 2000 .

8. Yang Y, Macleod V, Bendre M, et al: Heparanase promotes the spontaneous metastasis of myeloma cells to bone. Blood 105 : 1303-1309, 2005.

9. Baraz L, Haupt Y, Elkin M, Peretz T and Vlodavsky I: Tumor suppressor p53 regulates heparanase gene expression. Oncogene 25: 3939-3947, 2006.

10. Cohen I, Maly B, Simon I, et al: Tamoxifen induces heparanase expression in estrogen receptor-positive breast cancer. Clin Cancer Res 13: 4069-4077, 2007.

11. Wang F, Wang Y, Kim MS, et al: Glucose-induced endothelia heparanase secretion requires cortical and stress actin reorganization. Cardiovasc Res 87: 127-136, 2010.

12. Vitale FV, Rotondo S, Sessa E, et al: Low molecular weight heparin administration in cancer patients with hypercoagulability-related complications and carrying brain metastases: a case series study. J Oncol Pharm Pract 18: 10-16, 2011.

13. Gandhi NS, Freeman C, Parish CR and Mancera RL: Computational analyses of the catalytic and heparin-binding sites and their interactions with glycosaminoglycans in glycoside hydrolase family 79 endo- $\beta$-D-glucuronidase (heparanase) Glycobiology 22: 35-55, 2012.

14. Schroder M and Kaufman RJ: The mammalian unfolded protein response. Annu Rev Biochem 74: 739-789, 2005

15. Takada A, Miki T, Kuno A, et al: Role of ER stress in ventricular contractile dysfunction in type 2 diabetes. PLoS One 7: e39893, 2012.

16. Li N, Zoubeidi A, Beraldi E and Gleave ME: GRP78 regulates clusterin stability, retrotranslocation and mitochondrial localization under ER stress in prostate cancer. Oncogene: June 11, 2012 (Epub ahead of print).

17. Dong D, Ni M, Li J, et al: Critical role of the stress chaperone GRP78/BiP in tumor proliferation, survival, and tumor angiogenesis in transgene-induced mammary tumor development. Cancer Res 68: 498-505, 2008.

18. Weng WC, Lee WT, Hsu WM, Chang BE and Lee H: Role of glucose-regulated Protein 78 in embryonic development and neurological disorders. J Formos Med Assoc 110: 428-437, 2011.

19. Nasser NJ, Sarig G, Brenner B, et al: Heparanase neutralizes the anticoagulation properties of heparin and low-molecular-weight heparin. J Thromb Haemost 4: 560-565, 2006.

20. Liang Y, O'Driscoll L, McDonnell S, et al: Enhanced in vitro invasiveness and drug resistance with altered gene expression patterns in a human lung carcinoma cell line after pulse selection with anticancer drugs. Int J Cancer 111: 484-493, 2004.
21. Bruzzi P, Del Mastro L, Sormani MP, et al: Objective response to chemotherapy as a potential surrogate end point of survival in metastatic breast cancer patients. J Clin Oncol 23: 5117-5125, 2005.

22. Ilan NM, Elkin and Vlodavsky I: Regulation, function and clinical significance of heparanase in cancer metastasis and angiogenesis. Int J Biochem Cell Biol 38: 2018-2039, 2006.

23. Meirovitz A, Hermano E, Lerner I, et al: Role of heparanase in radiation-enhanced invasiveness of pancreatic carcinoma. Cancer Res 71: 2772-2780, 2011.

24. Nasser NJ: Heparanase involvement in physiology and disease. Cell Mol Life Sci 65: 1706-1715, 2008.

25. Theocharis AD, Skandalis SS, Tzanakakis GN and Karamanos NK: Proteoglycans in health and disease: novel roles for proteoglycans in malignancy and their pharmacological targeting. FEBS J 277: 3904-3923, 2010

26. Fan L, Wu Q, Xing X, Liu Y and Shao Z: Targeted silencing of heparanase gene by small interfering RNA inhibits invasiveness and metastasis of osteosarcoma cells. J Huazhong Univ Sci Technolog Med Sci 31: 348-352, 2011.

27. Vlodavsky I, Beckhove P, Lerner I, et al: Significance of heparanase in cancer and inflammation. Cancer Microenviron 5: 115-132, 2011.

28. Su R, Li Z,Li H, et al: Grp78 promotes the invasion of hepatocellular carcinoma. BMC Cancer 10: 20, 2010.

29. Scull CM and Tabas I: Mechanisms of ER stress-induced apoptosis in atherosclerosis. Arterioscler Thromb Vasc Biol 31: 2792-2797, 2011.

30. Nishitoh $\mathrm{H}$ : $\mathrm{CHOP}$ is a multifunctional transcription factor in the ER stress response. J Biochem 151: 217-219, 2012.

1. Huang KH, Kuo KL, Chen SC, et al: Down-regulation of glucose-regulated protein (GRP) 78 potentiates cytotoxic effect of celecoxib in human urothelial carcinoma cells. PLoS One 7: e33615, 2012

32. Feng X, Krishnan K, Richie DL, et al: HacA-independent functions of the ER stress sensor IreA synergize with the canonical UPR to influence virulence traits in Aspergillus fumigatus. PLoS Pathog 7: e1002330, 2011.

33. Fribley AM, Miller JR, Reist TE, Callaghan MU and Kaufman RJ: Large-scale analysis of UPR-mediated apoptosis in human cells. Methods Enzymol 491: 57-71, 2011.

34. Fiamoli V, Blatny J, Zapleta O, Kohlerova S and Janousova E: Treatment of deep vein thrombosis with continuous IV infusion of LMWH: a retrospective study in 32 children. Thrombosis 2011: 981497, 2011

35. Zhao H, Liu H, Chen Y, et al: Oligomannurarate sulfate, a novel heparanase inhibitor simultaneously targeting basic fibroblast growth factor, combats tumor angiogenesis and metastasis. Cancer Res 66: 8779-8787, 2006.

36. Basche M, Gustafson DL, Holden SN, et al: A phase I biological and pharmacologic study of the heparanase inhibitor PI-88 in patients with advanced solid tumors. Clin Cancer Res 12: 5471-5480, 2006.

37. Xu YZ, Zhu Y, Shen ZJ, et al: Significance of heparanase-1 and vascular endothelial growth factor in adrenocortical carcinoma angiogenesis: potential for therapy. Endocrine 40: 445-451, 2011.

38. Sanderson RD and Iozzo RV: Targeting heparanase for cancer therapy at the tumor-matrix interface. Matrix Biol 31: 283-284, 2012. 